\title{
The Boundedness of Bessel-Riesz Operators On Morrey Spaces
}

\author{
Mochammad Idris ${ }^{1, a)}$, Hendra Gunawan ${ }^{1}$, Janny Lindiarni ${ }^{1}$ and Eridani ${ }^{2}$ \\ ${ }^{1}$ Department of Mathematics, Institut Teknologi Bandung, Bandung 40132, Indonesia \\ ${ }^{2}$ Department of Mathematics, Airlangga University, Campus C, Mulyorejo, Surabaya 60115, Indonesia \\ ${ }^{\text {a)} C o r r e s p o n d i n g ~ a u t h o r: ~ m o c h i d r i s @ ~ @ s t u d e n t s . i t b . a c . i d ~}$
}

\begin{abstract}
In this paper, we shall discuss about Bessel-Riesz operators. Kurata et al. have investigated their boundedness on generalized Morrey spaces with weight. The boundedness of these operators on Lebesgue spaces and Morrey spaces will be reproved using a different approach. Moreover, we also find the norm of the operators are bounded by the norm of the kernels.
\end{abstract}

Keywords: Bessel-Riesz operators, Hardy-Littlewood maximal operator, Morrey spaces.

\section{INTRODUCTION}

Let $0<\gamma, 0<\alpha<n$ and define

$$
I_{\alpha, \gamma} f(x):=\int_{\mathbb{R}^{n}} K_{\alpha, \gamma}(x-y) f(y) d y
$$

for every $f \in L_{l o c}^{p}\left(\mathbb{R}^{n}\right)$, where $p \geq 1, K_{\alpha, \gamma}(x):=\frac{|x|^{\alpha-n}}{\left(1+|x|^{\gamma}\right.}, x \in \mathbb{R}^{n}$. Here, $K_{\alpha, \gamma}$ can be viewed as multiple of two kernels, $K_{\alpha, \gamma}(x)=J_{\gamma}(x) K_{\alpha}(x)$ for every $x \in \mathbb{R}^{n}$. In [1], $J_{\gamma}$ and $K_{\alpha}$ are known as Bessel kernel and Riesz kernel. So, $K_{\alpha, \gamma}$ is called Bessel-Riesz kernel and $I_{\alpha, \gamma}$ is called Bessel-Riesz operator.

For $\gamma=0$, we have $I_{\alpha, 0}=I_{\alpha}$ (is called fractional integral operator or Riesz potential [1]). Studies about $I_{\alpha}$ were started since 1920's. Hardy-Littlewood [2,3] and Sobolev [4] proved the boundedness of $I_{\alpha}$ on Lebesgue spaces through the inequality $\left\|I_{\alpha} f\right\|_{L^{q}} \leq C_{p}\|f\|_{L^{p}}$, for every $f \in L^{p}\left(\mathbb{R}^{n}\right), 1<p<\frac{\alpha}{n}$, and $\frac{1}{q}=\frac{1}{p}-\frac{\alpha}{n}$.

For $1 \leq p \leq q$, the (classical) Morrey space $L^{p, q}\left(\mathbb{R}^{n}\right)$ is defined by

$$
L^{p, q}\left(\mathbb{R}^{n}\right):=\left\{f \in L_{\text {loc }}^{p}\left(\mathbb{R}^{n}\right):\|f\|_{L^{p, q}}<\infty\right\},
$$

where $\|f\|_{L^{p, q}}:=\sup _{r>0, a \in \mathbb{R}^{n}} r^{n(1 / q-1 / p)}\left(\int_{|x-a|<r}|f(x)|^{p} d x\right)^{1 / p}$. We have an inclusion property for Morrey spaces $L^{q}\left(\mathbb{R}^{n}\right)=L^{q, q}\left(\mathbb{R}^{n}\right) \subseteq L^{p, q}\left(\mathbb{R}^{n}\right) \subseteq L^{1, q}\left(\mathbb{R}^{n}\right)$.

On Morrey spaces, Spanne [5] has shown that $I_{\alpha}$ is bounded form $L^{p_{1}, q_{1}}\left(\mathbb{R}^{n}\right)$ to $L^{p_{2}, q_{2}}\left(\mathbb{R}^{n}\right)$ for $1<p_{1}<q_{1}<\frac{n}{\alpha}$, $\frac{1}{p_{2}}=\frac{1}{p_{1}}-\frac{\alpha}{n}$, and $\frac{1}{q_{2}}=\frac{1}{q_{1}}-\frac{\alpha}{n}$. Furthermore, Adams [6] and Chiarenza-Frasca [7] obtained a stronger result.

Theorem 1 [Adams, Chiarenza-Frasca] If $0<\alpha<n$ then we have

$$
\left\|I_{\alpha} f\right\|_{L^{p_{2}, q_{2}}} \leq C_{p_{1}, q_{1}}\|f\|_{L^{p_{1}, q_{1}}},
$$

for every $f \in L^{p_{1}, q_{1}}\left(\mathbb{R}^{n}\right)$ where $1<p_{1}<q_{1}<\frac{n}{\alpha}, \frac{1}{p_{2}}=\frac{1}{p_{1}}\left(1-\frac{\alpha q_{1}}{n}\right)$, and $\frac{1}{q_{2}}=\frac{1}{q_{1}}-\frac{\alpha}{n}$.

Meanwhile, we have $\left|I_{\alpha, \gamma} f(x)\right| \leq\left|I_{\alpha} f(x)\right|$, for every $f \in L_{l o c}^{p}\left(\mathbb{R}^{n}\right)$. Using this inequality, $I_{\alpha, \gamma}$ is bounded on these spaces. In 1999, Kurata et. al [8] have proved that $W \cdot I_{\alpha, \gamma}$ is bounded on generalized Morrey spaces where $W$ is a multiplication operator. Here, we shall discuss about the boundedness of $I_{\alpha, \gamma}$ on Lebesgue spaces and Morrey spaces. We shall see the influence of $K_{\alpha, \gamma}$ for the boundedness of $I_{\alpha, \gamma}$. 